\title{
TU/e EmonOWEN

\section{Impact of interaction range and curvature on crystal growth of particles confined to spherical surfaces}

\section{Citation for published version (APA):}

Paquay, S., Both, G-J., \& Van Der Schoot, P. P. A. M. (2017). Impact of interaction range and curvature on crystal growth of particles confined to spherical surfaces. Physical Review E, 96(1), [012611].

https://doi.org/10.1103/PhysRevE.96.012611

DOI:

10.1103/PhysRevE.96.012611

Document status and date:

Published: 31/07/2017

\section{Document Version:}

Publisher's PDF, also known as Version of Record (includes final page, issue and volume numbers)

\section{Please check the document version of this publication:}

- A submitted manuscript is the version of the article upon submission and before peer-review. There can be important differences between the submitted version and the official published version of record. People interested in the research are advised to contact the author for the final version of the publication, or visit the $\mathrm{DOI}$ to the publisher's website.

- The final author version and the galley proof are versions of the publication after peer review.

- The final published version features the final layout of the paper including the volume, issue and page numbers.

Link to publication

\section{General rights}

Copyright and moral rights for the publications made accessible in the public portal are retained by the authors and/or other copyright owners and it is a condition of accessing publications that users recognise and abide by the legal requirements associated with these rights.

- Users may download and print one copy of any publication from the public portal for the purpose of private study or research.

- You may not further distribute the material or use it for any profit-making activity or commercial gain

- You may freely distribute the URL identifying the publication in the public portal.

If the publication is distributed under the terms of Article 25fa of the Dutch Copyright Act, indicated by the "Taverne" license above, please follow below link for the End User Agreement:

www.tue.nl/taverne

Take down policy

If you believe that this document breaches copyright please contact us at:

openaccess@tue.nl

providing details and we will investigate your claim. 


\title{
Impact of interaction range and curvature on crystal growth of particles confined to spherical surfaces
}

\author{
Stefan Paquay* \\ Department of Applied Physics, Eindhoven University of Technology, P.O. Box 513, 5600 MB Eindhoven, The Netherlands \\ and Physics Department, Brandeis University MS057, 415 South Street, Waltham, Massachusetts 02453, USA \\ Gert-Jan Both \\ Department of Applied Physics, Eindhoven University of Technology, P.O. Box 513, 5600 MB Eindhoven, The Netherlands \\ Paul van der Schoot \\ Department of Applied Physics, Eindhoven University of Technology, P.O. Box 513, 5600 MB Eindhoven, The Netherlands \\ and Instituut voor Theoretische Fysica, Universiteit Utrecht, The Netherlands
}

(Received 16 March 2017; revised manuscript received 26 June 2017; published 31 July 2017)

\begin{abstract}
When colloidal particles form a crystal phase on a spherical template, their packing is governed by the effective interaction between them and the elastic strain of bending the growing crystal. For example, if growth commences under appropriate conditions, and the isotropic crystal that forms reaches a critical size, growth continues via the incorporation of defects to alleviate elastic strain. Recently, it was experimentally found that, if defect formation is somehow not possible, the crystal instead continues growing in ribbons that protrude from the original crystal. Here we report on computer simulations in which we observe both the formation of ribbons at short interaction ranges and packings that incorporate defects if the interaction is longer-ranged. The ribbons only form above some critical crystal size, below which the nucleus is disk-shaped. We find that the scaling of the critical crystal size differs slightly from the one proposed in the literature, and we argue that this is because the actual morphology transition is caused by the competition between line tension and elastic stress, rather than the competition between chemical potential and elastic stress.
\end{abstract}

DOI: 10.1103/PhysRevE.96.012611

\section{INTRODUCTION}

Colloidosomes are droplets whose surfaces are densely packed with colloidal particles [1]. Confinement of the colloidal particles to the liquid-liquid interface minimizes the contact area between the two liquids. At high surface coverage, the colloids pack either in a disordered, glassy fashion or in an ordered, crystalline fashion [1-4]. In the former, particles are kinetically trapped. In the latter, the equilibrium packing of the colloids is determined by the interplay between the curvature of the droplet and the exact nature of the interaction between the colloids. This can give rise to various kinds of defect and defect organizations, producing grain boundary scars [5-7], pleats [8], and/or growth in ribbonlike shapes that emanate from isotropic domains without defects at the boundary between the two [9].

Grain boundary scars and pleats involve point defects in specific arrangements, where a point defect is a particle that has fewer or more than six nearest neighbors. Ribbons form when particles, for whatever reason, refuse to give up local hexagonal order. One such reason can be elastic stress, resulting from a short interaction range $[9,10]$. In macroscopic theory, this stress manifests itself in an additional elastic term in the free energy $[11,12]$. The experimentally observed ribbonlike crystals of Meng et al. show an initial, almost isotropic growth that, after a critical size is reached, transitions into the growth of ribbons protruding from the initial crystal [9]. Similarly, in phase-field crystal model calculations performed by Köhler et al., such structures are also found, confirming that growing

*Corresponding author: spaquay@brandeis.edu in ribbons indeed leads to a lower free energy [10]. Note that the crystal growth is continuous along the interface between the initial nucleus and the ribbon, i.e., there is no grain boundary between the two because the ribbon preserves the same lattice vectors.

The reason ribbons form can be understood from a microscopical picture. In a hexagonal crystal, point defects always have a larger potential energy than sixfold oriented particles because they cannot be at the optimal lattice site with respect to all of their neighbors. For a reasonably broad interaction well, this causes a slight increase in the potential energy that is not sufficiently punitive to destabilize such a defect.

For very sharp (brittle) potentials, however, the potential energy penalty for a point defect is so large that it destabilizes point defects completely. This means that the only crystal morphology that can form is hexagonal. Since wrapping a hexagonal lattice on a spherical surface is impossible without introducing defects, brittle potentials can only achieve this by introducing vacancies. Therefore, the tears in between ribbons, as seen in the experiments of Meng et al., can be thought of as regions in which the elastic penalty for crystal formation is prohibitively high.

Theoretical arguments for how the largest isotropic domain should scale with the Young's modulus and template radius were given in the aforementioned studies and are based on finding the optimal size of a circular crystal bent onto a spherical surface, which is, in this topology, bounded by an elastic energy penalty $[9,10]$. The theory predicts that ribbons form for a sufficiently short range of interaction, for example of a suitably parametrized Morse potential [13-15], and only then for sufficiently large domain sizes. For the classical nucleation 
theory proposed in these articles, this critical domain size follows from the competition between the chemical potential of the crystal and the elastic bending penalty.

We find by means of computer simulation that the range of the interaction potential between the particles indeed dictates whether or not ribbons form. For sufficiently large interaction ranges, ribbon formation is suppressed in favor of incorporating defects into the crystal. For shorter ranges of interaction, the critical size for ribbon formation reduces, which is consistent with the previous experimental and theoretical phase-field studies [9,10]. However, the scaling exponent we observe is slightly different, and it coincides with the scaling of the optimal width of a pure ribbon on a spherical surface, proposed more recently by Grason [16]. Interestingly, we do not observe the reentrance of point defects at the shortest ranges of attraction predicted by Grason [16]. This could be due either to our shortest range of attraction not being sufficiently short, or to large kinetic barriers between the ribbonlike structures and an isotropic crystal.

The remainder of this article is structured as follows. In Sec. II we present in detail classical nucleation theory for curved crystals, and we derive from them the scaling for the critical isotropic domain size proposed by Meng et al. and Köhler et al. [9,10], as well as our critical isotropic domainsize scaling based on a hybrid crystal with a disklike and a rectangular region, which coincides with the one proposed by Grason [16]. In Sec. III we discuss the simulation and analysis methods. In Sec. IV we discuss our computational findings and show that we indeed observe a critical domain size and extract its scaling relations with respect to the potential range and the radius of curvature. Finally, in Sec. V we underline the most important implications of our findings.

\section{CLASSICAL NUCLEATION THEORY ON CURVED SURFACES}

\section{A. Flat plane}

Macroscopically, the formation of a two-dimensional crystal on a flat surface is described by classical nucleation theory (CNT) [17]. Although this theory is well known, we introduce it here because we extend later it with elastic terms to describe crystal formation on curved surfaces. Furthermore, it will serve as the basis for our two-stage model for ribbon growth, which we introduce in Sec. II C. In CNT the thermodynamic driving force toward crystal formation is opposed by a surface tension that in two dimensions is a line tension. If we assume the line tension to be invariant to the locally exposed crystal plane, the free energy of a crystal nucleus is given by $\Delta G=N \Delta \mu+\gamma L$, where $N$ is the number of particles in the crystal, $\Delta \mu<0$ is the chemical potential difference between the crystal phase and the surrounding liquid or gas, $L$ is the crystal circumference, and $\gamma$ is the line tension $[17,18]$.

If we assume a circular crystal, we have $L=\pi a$, with $a$ the diameter of the crystal so that $\Delta G_{c}=N \Delta \mu+\pi a \gamma$. Note that actual two-dimensional crystals tend to form hexagonal nuclei instead because the underlying interaction potential encourages hexagonal bond order that induces anisotropic growth [17-19]. However, since the area of a circle and hexagon scale as the number of particles squared, and for the purpose of extracting scaling laws it is a good approximation. Limitless growth of the drop occurs if $\Delta G<0$, i.e., if $N>$ $-\pi a \gamma / \Delta \mu$. Of course, $a=2 \sqrt{N / \pi \rho}$ depends on $N$ through the equilibrium particle surface density $\rho$ of the crystal. In other words, limitless growth occurs for $N>4 \pi \gamma^{2} /(\Delta \mu)^{2} \rho$. Converting the expression for the free energy to the circle diameter $a$ leads to

$$
\Delta G_{c}=\rho \Delta \mu \pi a^{2} / 4+\gamma \pi a .
$$

Ribbonlike crystals are better described by a rectangle with a circumference $A=2(w+l)$ and a particle number $N=$ $\rho w l$, leading to

$$
\Delta G_{r}=\rho \Delta \mu w l+2 \gamma(w+l) .
$$

Unlike the circle, the ribbon has two parameters $w$ and $l$, and the optimal free energy has $w=l$, as this gives the largest area for a given circumference. In the flat plane, a circlelike shape will always be preferred over a ribbonlike shape. This can be seen by comparing Eqs. (1) and (2) under the constraint that they have equal areas, $w l=\pi a^{2} / 4$. Their difference is then simply the difference of the line tension terms, $\gamma[\pi a-2(w+$ $\left.\left.\pi a^{2} / 4 w\right)\right]$, which, if we substitute the optimal $w=\sqrt{\pi} a / 2$, is always negative. Therefore, not surprisingly, in a flat plane, a ribbon is always destabilized with respect to a circle. However, as becomes clear later, this trivial conclusion no longer holds on a curved surface.

To see whether or not a ribbon is more likely to be nucleated than a circle, we consider the kinetic barrier heights. For a circle, the maximum in $\Delta G_{c}$ corresponds to a critical diameter $a=-2 \gamma / \rho \Delta \mu$ and is equal to $-\pi \gamma^{2} / \rho \Delta \mu$, where we recall that $\Delta \mu<0$. For the ribbon the maximum is located at $l=$ $w=-2 \gamma / \rho \Delta \mu$ and has a value of $-4 \gamma^{2} / \rho \Delta \mu$. Therefore, in the flat plane, a ribbonlike crystal is also less likely to nucleate, as evidenced by a higher nucleation barrier in the free energy. This means that in a two-dimensional plane, a disklike crystal will always be favored over a ribbon-shaped one, both thermodynamically and kinetically.

\section{B. On a sphere}

The above picture changes if we consider crystals constrained to a spherical surface of radius $R$. In this case, an additional energy enters the free energy that takes into account the elastic cost of bending the crystal to accommodate the curved template $[11,12]$. First consider again the circular crystal. To make the analysis more straightforward, we scale the free energy to the spherical surface area, $4 \pi R^{2}$, times Young's modulus $Y$. This leads to a reduced unit $\eta:=a / R$ and a dimensionless free energy [9-12],

$$
\Delta g_{c}=\frac{1}{4 \pi}\left[\frac{\rho \Delta \mu \pi}{4 Y} \eta^{2}+\frac{\pi \gamma}{R Y} \eta+\frac{\pi}{24576} \eta^{6}\right] .
$$

Although the elastic term does not influence the kinetic barrier height or critical diameter significantly [20], it does influence the thermodynamic stability of the circular crystal. For very large $Y$, the elastic term dominates to such an extent that the local minimum in the free energy that occurs for sizes larger than the critical nucleus size becomes larger than $0[10,20,24]$. In that case, $\Delta g_{c} \geqslant 0$, and the formation of a circular crystal is thermodynamically suppressed. 
Furthermore, for $Y>0$, there is now an optimal circle diameter that minimizes the free energy. Under the assumption $\gamma \ll R Y$, this minimum is located at $\eta=$ $(-6144 \rho \Delta \mu / Y)^{1 / 4}:=\eta_{0}$. This value is associated with the critical isotropic crystal size at which growth transitions to a ribbon, as circular domains larger than this radius have a higher free energy $[9,10]$. We shall refer to this scaling as the optimal circle scaling. However, as long as $\Delta g_{c}<0$, the circle can in principle continue to grow, for example if there is not a sufficient amount of material to nucleate a second, stable disklike cluster.

Unlike in the flat plane, on a sphere a ribbon-shaped crystal can be more stable than a circle due to a different scaling of the elastic energy with the crystal dimensions. The dimensionless free energy for the ribbon on a spherical surface is given in terms of dimensionless width $\omega=w / R$ and length $\lambda=l / R$ by $[11,12]$

$$
\Delta g_{r}=\frac{1}{4 \pi}\left[\frac{\rho \Delta \mu}{Y} \omega \lambda+\frac{2 \gamma}{R Y}(\omega+\lambda)+\frac{\omega^{5} \lambda}{640\left(1-v^{2}\right)}\right],
$$

where $v$ is the Poisson ratio of the material. Note that in this expression, $\rho \Delta \mu \lambda+2 \gamma / R$ has to be negative, and hence it only holds for $\rho \Delta \mu<-2 \gamma / R \lambda=-2 \gamma / L$.

The reason a ribbon can be stabilized over an isotropic crystal on a curved surface is because although the elastic strain scales with $\omega^{5}$, it only scales linearly with $\lambda$. This leads to a different scaling with the total crystal area, which becomes obvious when the free energy of the ribbon is expressed in terms of the width $\omega$ and dimensionless area $A / R^{2}:=\zeta=\omega \lambda$,

$$
\Delta g_{r}=\frac{1}{4 \pi}\left[\left(\frac{\rho \Delta \mu}{Y}+\frac{\gamma}{R Y} \frac{2}{\omega}+\frac{\omega^{4}}{640\left(1-v^{2}\right)}\right) \zeta+\frac{\gamma}{R Y} 2 \omega\right] .
$$

This free energy can in principle be optimized with respect to $\omega$ to obtain an optimal ribbon width for a given area:

$$
4 \pi \frac{\partial \Delta g_{r}}{\partial \omega}=\left[-\frac{\gamma}{R Y} \frac{2}{\omega^{2}}+\frac{\omega^{3}}{160\left(1-v^{2}\right)}\right] \zeta+2 \frac{\gamma}{R Y}=0 .
$$

Because the lowest-order term $-2 \gamma / R Y \zeta \omega^{2}$ is negative, there will always be some $\omega$ that optimizes Eq. (5).

For the ribbon to be stable at some point, the total free energy should be negative, i.e., $\Delta g_{r}<0$. Since $2 \omega \gamma / R Y>0$, the ribbon can only be stable when

$$
\left[\left(\frac{\rho \Delta \mu}{Y}+\frac{\gamma}{R Y} \frac{2}{\omega}+\frac{\omega^{4}}{640\left(1-v^{2}\right)}\right)\right] \zeta<-2 \omega \frac{\gamma}{R Y} .
$$

Since $0 \leqslant \zeta \leqslant 1$, this condition can only be satisfied if at least

$$
\frac{\rho \Delta \mu}{Y}+\frac{\gamma}{R Y} \frac{2}{\omega}+\frac{\omega^{4}}{640\left(1-v^{2}\right)}+2 \omega \frac{\gamma}{R Y} \leqslant 0 .
$$

The equality can only be achieved for sufficiently small $\rho \Delta \mu / Y$ and $\gamma R Y$, i.e., at sufficiently large Young's moduli. When this inequality holds, a ribbon can in principle be indefinitely large and still have a negative free energy, unlike an isotropic crystal.

However, the kinetic barrier associated with a ribbon is always larger than that of a circle. Under the assumption that the elastic contribution is negligible for crystals smaller

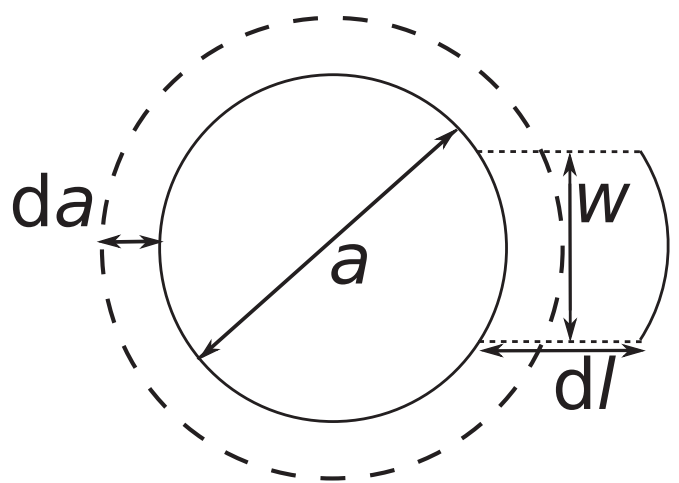

FIG. 1. Two possible modes of growth from a circular nucleus of diameter $a$. Either growth continues along an infinitesimal diameter increase $d a$, or as a ribbonlike structure of width $w$ and length $d l$ protruding out of the circular nucleus.

than the critical nucleus size, we find that the barrier height, i.e., the maximum in $\Delta g$, is located at $\eta_{b}=-2 \gamma / \rho \Delta \mu R$ for the circle and at $\omega_{b}=\eta_{b}, \zeta_{b}=\omega_{b}^{2}$ for the ribbon. The corresponding free energies are $\Delta g_{c}^{b}=-\gamma^{2} / 4 \rho \Delta \mu R^{2}$ and $\Delta g_{r}^{b}=-\gamma^{2} / \pi \rho \Delta \mu R^{2}$. Hence, the kinetic barrier for formation of a circle is lower than that of a ribbon by a factor of $\pi / 4$.

This implies that, while under certain conditions a ribbonshaped crystal has a lower free energy, it is less likely to be nucleated. A more likely scenario that leads to the formation of ribbonlike structures is therefore a two-stage nucleation, in which the ribbon grows out of a disklike nucleus. In such an event, the crystal has the kinetic energy barrier of a circular shape and initially grows as such. However, at some point, the free energy decrease for continued growth as a circle is smaller than that for growth as a ribbon, and hence the crystal continues growth into ribbonlike structures. Such a growth pathway is also more consistent with the experimental results [9] and crystal phase-field calculations [10]. In Sec. IIC we present a free energy for such a growth type.

\section{Two-stage nucleation theory on a sphere}

In Sec. II we argue that the free energy of a disklike crystal is, under certain conditions, lower than that of a ribbon. Hence, the formation of a pure ribbonlike crystal is not probable, as ribbons smaller than the transition size are destabilized with respect to isotropic crystals with equal area. This motivates us to investigate the possibility of a two-stage nucleation, meaning that a ribbon-shaped structure grows out of a preexisting, circular nucleus. For this to happen, the free energy gain due to a small area increment in the shape of a ribbon should be smaller than that due to an equal area increment in the shape of the already existing circle. Both growth modes are sketched in Fig. 1.

From the typical crystal morphologies presented by Meng et al. [9] and Köhler et al. [10], it appears that the ribbons grow out of the initial crystals without distorting the lattice vectors. Therefore, we assume there is no line tension associated with the circle-ribbon interface. We furthermore assume that the line tension associated with the width of the ribbon is the same as that of the previously exposed circular rim that is now 
covered by the ribbon. This means there is no line tension term associated with the width, because it is already accounted for by the original line tension term. With these assumptions, the total free energy of the hybrid crystal becomes

$$
\begin{aligned}
\Delta g_{c+r}= & \frac{1}{4 \pi}\left[\frac{\rho \Delta \mu}{Y}\left(A_{c}+A_{r}\right)+\frac{\gamma}{R Y}\left(\sqrt{4 \pi A_{c}}+2 \frac{A_{r}}{\omega}\right)\right. \\
& \left.+\frac{A_{c}^{3}}{384 \pi^{2}}+\frac{\omega^{4} A_{r}}{640\left(1-v^{2}\right)}\right],
\end{aligned}
$$

where $A_{r}$ is the dimensionless ribbon area $\omega \lambda$, and $A_{c}$ is the circle area $\pi \eta^{2} / 4$. The free-energy change of the crystal $d \Delta g_{r+c}$ of growing purely as a circle can be determined by expanding $\Delta g_{c+r}$ in a Taylor series around $A_{c}$ for $A_{r}=0$, leading to

$$
\begin{aligned}
d \Delta g_{c+r} & =\left(\frac{\partial \Delta g_{c+r}}{\partial A_{c}}\right) d A \\
& =\frac{d A}{4 \pi}\left[\frac{\rho \Delta \mu}{Y}+\frac{\gamma}{R Y} \sqrt{\frac{\pi}{A_{c}}}+\frac{A_{c}^{2}}{128 \pi^{2}}\right] .
\end{aligned}
$$

To determine the free-energy change of growing as a ribbon, we expand $\Delta g_{c+r}$ in terms of $A_{r}$ for fixed $\omega$ and $A_{c}$, leading to

$$
\begin{aligned}
d \Delta g_{c+r} & =\left(\frac{\partial \Delta g_{c+r}}{\partial A_{r}}\right) d A \\
& =\frac{d A}{4 \pi}\left[\frac{\rho \Delta \mu}{Y}+\frac{2 \gamma}{R Y \omega}+\frac{\omega^{4}}{640\left(1-v^{2}\right)}\right] .
\end{aligned}
$$

The width that gives the optimal free-energy change follows from optimizing $d \Delta g_{c+r}$ with respect to $\omega$, which leads to $\omega=\left[320\left(1-v^{2}\right) \gamma / R Y\right]^{1 / 5}$.

In other words, the free-energy change due to continued growth as a circle from an area $A_{c}$ with increment $\Delta A$ is given by $\left(\partial \Delta g_{c+r} / \partial A_{c}\right) \Delta A$, and the free-energy change due to growth as a ribbon out of a circular nucleus is given by ( $\left.\partial \Delta g_{c+r} / \partial A_{r}\right) \Delta A$. Therefore, the difference between Eqs. (7) and (8), $\Delta \Delta g$, indicates whether continued growth favors a ribbon or a circle,

$$
\begin{aligned}
\Delta \Delta g & :=\left(\frac{\partial \Delta g_{c+r}}{\partial A_{c}}-\frac{\partial \Delta g_{c+r}}{\partial A_{r}}\right) \Delta A \\
& =\frac{\Delta A}{4 \pi}\left[\frac{\gamma}{R Y}\left(\sqrt{\frac{\pi}{A_{c}}}-\frac{2}{\omega}\right)+\frac{A_{c}^{2}}{128 \pi^{2}}-\frac{\omega^{4}}{640\left(1-v^{2}\right)}\right] .
\end{aligned}
$$

The absence of $\Delta \mu$ in this expression makes it clear that it is the interplay between the line tension and the elastic stress, rather than between the elastic stress and the chemical potential, that determines the morphology, as previously also discussed by Grason [16].

Continued growth as a ribbon is preferred under two conditions. First of all, it is necessary that $\Delta \Delta g>0$, as only then is the free-energy gain for growing as a ribbon preferred over growing as a circle. Secondly, it is required that $\Delta g_{c+r}<0$, as otherwise the formation of any type of crystal at all is destabilized. Finally, although not technically a necessity for the initial onset of ribbon formation, we check whether or not $\partial \Delta g / \partial A_{r}<0$. If this is the case, the crystal can grow indefinitely as a ribbon, since continued growth as a ribbon will lead to a further decrease in the free energy.

To determine the critical area at which the transition takes place, we first numerically determine for a range of $\gamma / R Y$ and $\rho \Delta \mu$ the roots of $\Delta \Delta g$, i.e., the points that satisfy the first criterion. Then, we determine whether or not $\Delta g_{c+r}<0$ and what the sign of $\partial \Delta g_{c+r} / \partial A_{r}$ is. We find that $\Delta g_{c+r}<0$ is only satisfied at the roots of Eq. (9) for significantly large ratios for $|\rho \Delta \mu R / \gamma|$. In particular, we find that a ratio of at least 6 is required to obtain a transition at an area $A_{c}<0.6$ that satisfies all criteria, corresponding to $\gamma / R Y=2 \times 10^{-4}$ and $\rho \Delta \mu / Y=12 \times 10^{-4}$. Furthermore, although increasing Young's modulus leads to a decrease in the critical area at which $\Delta \Delta g=0$, it also leads to a higher free energy, occasionally such that $\Delta g_{c+r}>0$ at the transition. Hence, although larger Young's moduli favor ribbon formation, they will only actually form at a proportional decrease in the chemical potential.

To determine how the elastic cost influences the area at which the transition occurs, we determine how the critical isotropic area scales with Young's modulus for various ratios of the chemical potential and line tension terms. In Fig. 2 we plot our findings, which clearly show that the critical area scales as $A_{c} \sim(\gamma / R Y)^{2 / 5}$ and hence the diameter of the largest isotropic domain size scales as $\eta \sim(\gamma / R Y)^{1 / 5}$. Note that all points collapse on the same curve given by $A_{c}=$ $23.6594(\gamma / R Y)^{0.4}$. The relative standard errors in the linear regression are $1.3 \times 10^{-4} \%$ for the prefactor and $3.5 \times 10^{-5} \%$ for the exponent. Both the prefactor and the scaling exponent are independent of $\rho \Delta \mu / Y$.

We notice a clear breakdown in this scaling for $\gamma / R Y>$ $2 \times 10^{-4}$. In this regime, the line tension is prohibitively large and suppresses ribbon formation. There is another breakdown for sufficiently small $\gamma / R Y$ in combination with small $\rho \Delta \mu / Y$. In this regime, the chemical potential is not negative enough to compensate for the combined costs of bending and the line tension; in other words, there is no negative free energy $\Delta g_{c+r}<0$ anymore.

Hence, our analysis suggests that, if the formation of ribbons indeed proceeds as a two-stage, heterogeneous nucleation of a ribbonlike structure on a preexisting, circular nucleus, the normalized area of this circular nucleus should scale as $A_{c}=\pi a^{2} / 16 \pi R^{2} \sim(\gamma / R Y)^{2 / 5}$, where $a$ is the diameter of the circular crystal and $R$ is the radius of the template. This is different from the scalings previously proposed $[9,10]$, in which the transition area is associated with the minimum in $\Delta g_{c}$, which, in the limit of negligible line tension, scales as $A_{c} \sim(\rho \Delta \mu / Y)^{1 / 4}$.

The actual scaling of the transition area can be obtained from computer simulations by determining the largest isotropic domain size as a function of the spherical template radius, the chemical potential, the line tension, and Young's modulus, as is described in the main text and in the previous sections. We now describe the simulation methods we used to determine this scaling.

\section{METHODS}

We perform Langevin dynamics (LD) simulations of $N$ point particles constrained to a spherical surface with radius 

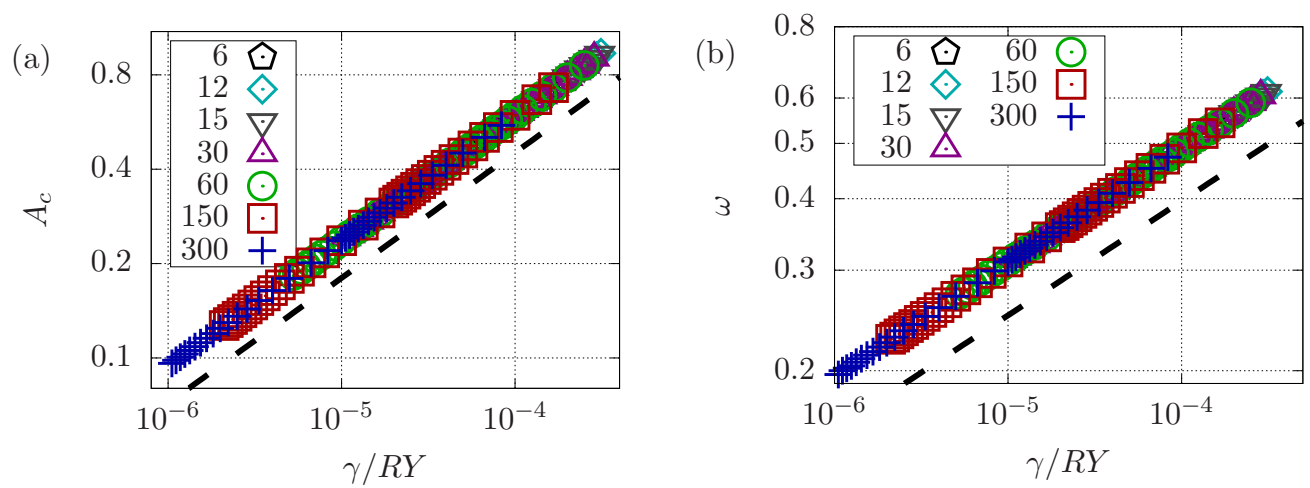

FIG. 2. Scaling of the isotropic domain area (a) and smallest ribbon width (b) at which growth transitions from a disklike crystal to a ribbon as a function of the dimensionless line tension $\gamma / R Y$. The legend indicates varying $\rho \Delta \mu R / Y$. Note that all points collapse onto the same curve. For smaller $\rho \Delta \mu R / Y$ there is no ribbon formation for smaller $\gamma / R Y$ because the free energy is no longer negative. The dashed line indicates the scaling predicted by theory, $A_{c} \sim(\gamma / R Y)^{2 / 5}$ (a) and $\omega \sim(\gamma / R Y)^{1 / 5}$ (b).

$R$, using the LAMMPS program and a specialized RATTLE algorithm [21-23]. The particle number $N$ we couple to the template radius as $N r_{0}^{2} / 16 R^{2}=0.4$, such that if we associate an area $\pi r_{0}^{2} / 4$ with each particle of diameter $r_{0}$, we have the same area coverage $\phi=0.4$ for all template radii. Initially, the particles are in nonoverlapping, random positions on the spherical surface.

We apply a Langevin thermostat with an arbitrary damping time $\tau_{L}$ to the particles to keep the system at a constant temperature $T$ and to make the particles undergo Brownian motion. $\tau_{L}$ is the time it takes for the velocity autocorrelation function of a particle to decay to $1 / e$ of its initial value, and it is our reference time unit that in effect measures the ratio of the particle's mass and the friction constant. For the interaction potential between particle pairs, we use a truncated and shifted Morse potential $U(r)$,

$$
U(r)=\left[U_{M}(r)-U_{M}\left(r_{c}\right)\right] H\left(r_{c}-r\right),
$$

where $r$ is the three-dimensional Cartesian interparticle distance, $r_{c}$ is the cutoff distance, $H\left(r_{c}-r\right)$ is the Heaviside step function that is 1 if $r_{c}-r>0$ and 0 otherwise, and $U_{M}(r)$ is the original Morse potential,

$$
U_{M}(r)=\epsilon\left[e^{2 \alpha\left(r-r_{0}\right)}-2 e^{\alpha\left(r-r_{0}\right)}\right],
$$

with a well depth $\epsilon$, equilibrium spacing $r_{0}$, and shape parameter $\alpha$. Throughout the remainder of this article, we use $r_{0}$ as the reference length unit and $\epsilon$ as the reference energy unit.

To encourage the growth of a single crystal, we lightly tether the particles to the top of the spherical surface $(x, y, z)=$ $(0,0, R)$ with a harmonic spring with spring constant $\kappa=$ $2.5 \epsilon / r_{0}^{2}$. We initialize the structure with this spring in place for $N_{s}$ time steps at a temperature of $k_{B} T=0.3 \epsilon$, after which we remove the springs. We then equilibrate for another $N_{s}$ steps while linearly ramping down the temperature from $k_{B} T=0.3 \epsilon$ to $0.25 \epsilon$. We verified that a longer ramping time had no influence on the formed structures. After this annealing phase, we sample for $N_{s}$ time steps at $k_{B} T=0.25 \epsilon$. We analyze snapshots that are $5 \tau_{l}$ apart in time and average over them to obtain good statistics. For $\alpha=40 / r_{0}$, a smaller time step of $0.001 \tau_{L}$ was required for numerical stability, and we set $N_{s}=25 \times 10^{6}$ to guarantee proper equilibration, while for the other values of $\alpha$ we used $N_{s}=250 \times 10^{3}$ and a time step size of $0.005 \tau_{L}$.

The parameter $\alpha$ sets how sharp the potential is peaked around its minimum at $r_{0}$, as the effective spring constant $\kappa$ is given by $\kappa=\partial^{2} U(r) /\left.\partial r^{2}\right|_{r_{0}}=2 \alpha^{2} \epsilon$. See Fig. 3 for a graph comparing the Lennard-Jones and Morse potentials for various values of $\alpha$. If we ignore the impact of multibody interactions, the Young's modulus scales linearly with $k$. This implies that a larger $\alpha$ leads to a larger Young's modulus [24]. Therefore, in our computer simulations we can use the parameter $\alpha$ to influence the Young's modulus of our crystal. By studying the particle vibrations about their lattice site, however, we see that the pair approximation is not very accurate. Hence, we rely on the actual vibrations to estimate the effective spring constant. See Appendix A for a detailed description of the analysis.

For our crystals, it turns out that effective spring constants $\kappa$ scale significantly less strongly with $\alpha$ than predicted by the harmonic approximation $\kappa \sim \alpha^{2}$. We obtain scalings between $\kappa \sim \alpha^{1.3}$ and $\kappa \sim \alpha^{1.6}$, with an average exponent of $(1.52 \pm$ $0.08)$. The deviation from the pair potential approximation could be due either to collective effects that are obviously

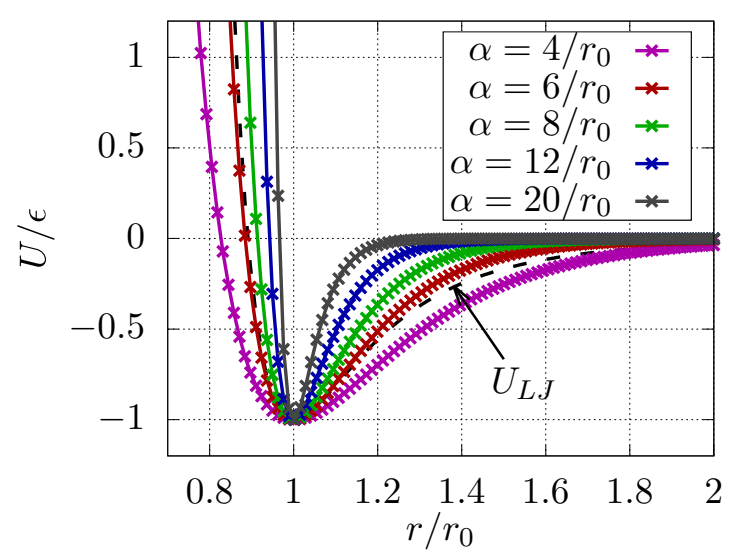

FIG. 3. Comparison of Morse (colored lines, symbols) and Lennard-Jones (dashed black) potentials. Increasing $\alpha$ leads to a sharper well for the Morse potential. For $\alpha=6 / r_{0}$ the harmonic approximation for the Morse potential equals that of the LennardJones potential. 
not included in any pairwise approximation, or to the nonzero temperature in our simulations, which influences the elastic properties [25]. This is not a complete surprise, as hard sphere crystals have an effective spring constant that follows entirely from many-body effects [26]. This shows that it is critical to measure Young's modulus or at least the effective spring constant of the material at hand rather than relying on the harmonic approximation when performing Langevin dynamics simulations. With the effective spring constant determined, we still need methods to determine the scaling of the chemical potential and the largest isotropic domain size with $\alpha$.

For larger values of $\alpha$ at which the crystal forms ribbons, most of the direct interaction is governed by nearest-neighbor interactions, as can be inferred from Fig. 3. Because of this, we assume the line tension arises predominantly from the edge particles having fewer than six bonds. In this case, we expect that the line tension and the chemical potential scale similarly with $\alpha$, and we only have to determine the scaling of $\Delta \mu$ with $\alpha$.

To determine the chemical potential, we have to determine the free-energy difference between a crystal consisting of $N$ Morse particles and that same crystal in which one of the bulk particles is changed to an ideal gas particle. This is problematic because an ideal gas particle tends to explore all of the spherical surface, while the Morse particle tends to stay near its lattice site. Therefore, we instead determine the free-energy difference associated with transforming one Morse particle into an "Einstein particle," that is, a particle that is tethered to its lattice site by a harmonic spring.

As a reference Morse particle, we take the particle that has the largest minimum distance to the edge particles. By applying Bennett's acceptance ratio [27] to the case of $N$ Morse particles and the case in which we transform this reference particle into an Einstein particle, we obtain free-energy differences that we can directly relate to the excess chemical potential. In Appendix B, a more detailed description of the procedure is presented. The found scaling is $\Delta \mu \sim \alpha^{(-0.53 \pm 0.01)}$. Note that the free-energy difference goes through an appreciable range as a function of $\alpha$, as we find $\Delta \mu \approx-0.7 / \epsilon$ for the largest $\alpha r_{0}=40$, which is a third of that of $\Delta \mu \approx-2.2 / \epsilon$ for the smallest $\alpha r_{0}=4$. It is thus important to take this scaling into account when assessing the scaling for the largest isotropic domain size at which the growth transitions to ribbons.

With the scalings for the effective spring constant $\kappa$ and hence Young's modulus $Y$ and that of the chemical potential $\Delta \mu$ and line tension $\gamma$ determined, we can now determine how the largest isotropic domain sizes scale as a function of the aforementioned parameters, provided that we can extract what the largest isotropic domain size is. We describe our approach now in brief.

Finding the largest isotropic domain size involves identifying the edge of what constitutes a circular domain and extracting the distance from that edge to the center of the domain, which can be reasonably approximated by finding the particle that is the farthest away from all edge particles. We calculate for all particles the shortest distance to the edge. The particle that is the farthest away from the edge we then consider to be the center of an isotropic domain, and we take its distance from the edge to be the radius of the largest circular (a)

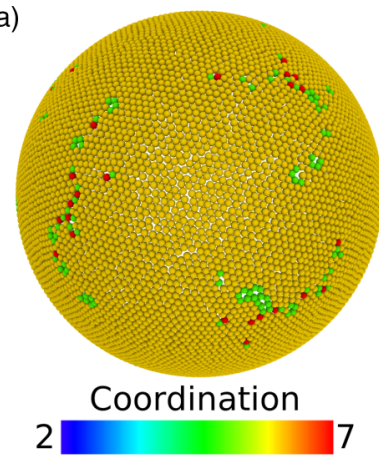

(c)

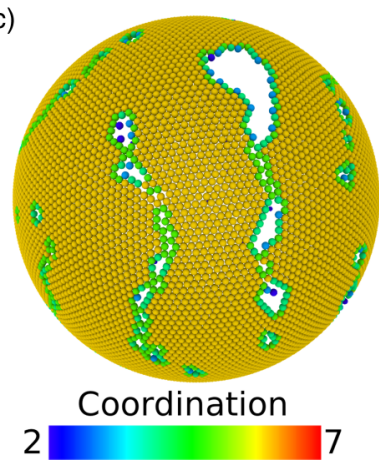

(b)

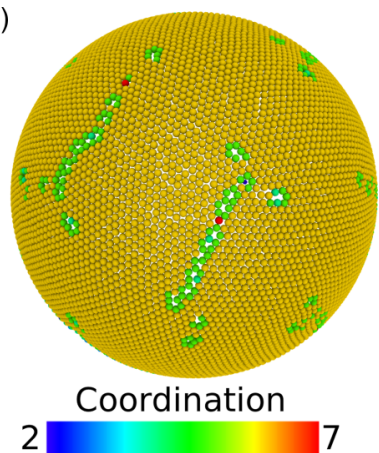

(d)

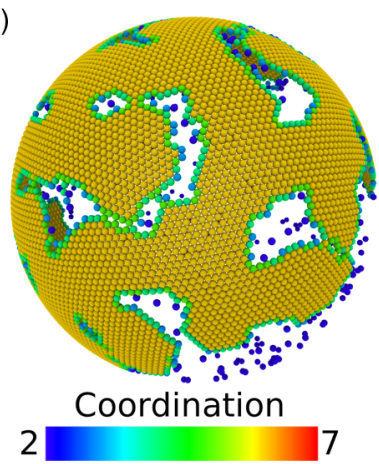

FIG. 4. Top view of typical crystals obtained from the simulation protocol at an area coverage of $40 \%$. The figures correspond to $R / r_{0}=30$ and $\alpha r_{0}=4$ (a), 8 (b), 16 (c), and 32 (d). Color codes for the number of nearest neighbors (coordination). Images rendered by Ovito [28]. The back of the spherical template is not covered except by particles in a gaseous phase that forms for $\alpha r_{0} \geqslant 16$. Note that for $\alpha r_{0}=4$ the crystal incorporates dislocations and point defects in the structure, as well as some entropically excited vacancies. For $\alpha r_{0} \geqslant 8$ the only defects occur as tears and holes between patches of a hexagonal lattice.

domain. Note that this particle is the same as the one for which we determine the chemical potential.

Incidentally, the largest isotropic domain size also gives a good indication of the transition from incorporated point defects to ribbons, as it scales differently with the range parameter $\alpha$ when the crystal incorporates point defects, as we shall see in Sec. IV. We perform all of the aforementioned analysis via postprocessing, according to the procedure described in Appendix C. With all the methods discussed, we now present our results.

\section{RESULTS}

In Fig. 4 we show typical crystal structures we observe as a function of the range parameter $\alpha$. The figures show the top half of the spherical template that is covered with particles. For $\alpha r_{0}=4$, the crystal incorporates various defects, predominantly dislocations [Fig. 4(a)], although there are some small, entropically excited vacancies. At around $\alpha r_{0}=$ 8 , a transition from incorporated defects to hexagonal packings occurs. From this point on, the defects are only located at the edge or around holes [Fig. 4(b)]. Increasing $\alpha$ further to $\alpha r_{0}=16$ [Fig. 4(c)] and $\alpha r_{0}=32$ [Fig. 4(d)] leads to a clear formation of ribbonlike structures separated by larger 
(a)

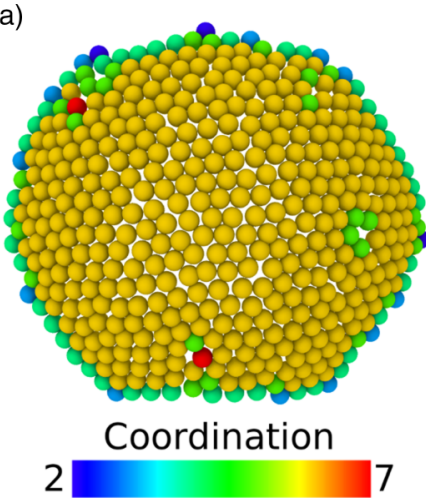

(b)

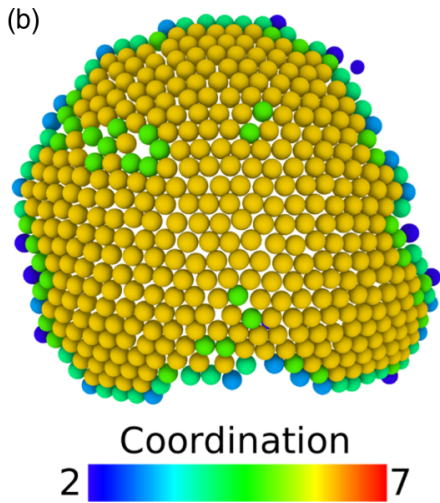

(c)

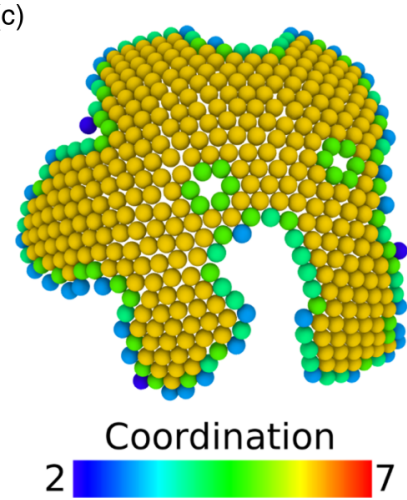

FIG. 5. Top view of crystals illustrating the transition from incorporated defects at $\alpha r_{0}=4$ (a) through an intermediate at $\alpha r_{0}=6$ (b) to a predominantly branched structure at $\alpha r_{0}=8$ (c) for 450 Morse particles interacting with varying $\alpha$ at an area coverage of $12.5 \%$.

tears. Furthermore, the width of the ribbons reduces, which is qualitatively consistent with the scalings presented by Meng et al., Köhler et al., and Grason [9,10,16]. Note that because of the entropically excited vacancies for small $\alpha$, we do not find structures that only contain disclinations or dislocations as defects, and hence the exact onset of ribbon formation is difficult to pinpoint.

The structures we obtain fluctuate significantly during the annealing phase. Even for the lower temperature at which we sample, there are still rearrangements of particles at the crystal edges. This makes us confident that the structures we sample are well-equilibrated and not kinetically trapped. The same simulation protocol applied to a flat plane leads to only hexagonal crystals, which is another indication that our structures are not kinetically trapped.

From Fig. 5 we see that at larger $\alpha$ the effective temperature of the particles appears to have increased slightly, in the sense that more particles appear in a gaslike phase on the side of the template that is not covered by the crystal. The motion of the particles inside the crystal, however, diminishes due to the increased steepness of the potential well depth, although there are still edge rearrangements.

Our crystals are reminiscent of the ones observed in simulations by Cong et al. [29]. For $\alpha r_{0} \geqslant 8$, we observe hexagonal patches separated by tears and holes, i.e., ribbons. Below that, the crystal incorporates pleats and point defects, which are reminiscent of those observed experimentally [5-8]. Hence, as shown experimentally by Meng et al. and consistent with the phase-field crystal calculations of Köhler et al., ribbons only form when the elastic strain, regulated by the range of the potential, prevents the formation of point defects $[9,10]$. Interestingly, we do not seem to observe the fourfold branching found in the phase-field crystal model, possibly due to the thermal fluctuations in our simulations, which are absent in the phase-field calculations.

In Fig. 5 we show an illustrative example of the difference between ribbons and incorporated point defects, obtained by applying the protocol of Sec. III to $N=450$ particles on a template of radius $R=15 r_{0}$ for $\alpha r_{0}=4,6$, and 12 . This example is obtained at an area coverage to $12.5 \%$ to illustrate more clearly the different structures. For $\alpha r_{0}=4$, the crystal is roughly circular and incorporates some defects. For $\alpha r_{0}=6$, we see the onset of a ribbon that is branching out of the initial bulk while there are still defects in the original nucleus as well. Hence, there appears to be a region where there is "coexistence" between incorporated defects and ribbon formation. For larger $\alpha r_{0}=8$, we see clear branching, and the defects in the bulk are significantly reduced.

We now analyze the size of the largest isotropic domain. All simulation results, from which the largest isotropic domain size is extracted below, correspond to a larger area coverage of $40 \%$ rather than the $12.5 \%$ that served as an illustration of the morphology in Fig. 5. First we consider the scaling of the largest isotropic domain size $a$ with the spherical template radius $R$. Figures 6(a) and 6(b) reveal that $a$ scales sublinearly with $R$. Fitting a power law reveals the scaling exponent to be $(0.83 \pm 0.06)$, which is inconsistent with the scaling proposed by Meng et al. and Köhler et al. [9,10]. It does match the scaling of the optimal ribbon width proposed by Grason [16], as well as our own theory based on heterogeneous nucleation of a ribbonlike structure on a disklike nucleus, presented in Sec. II. The reason for this is that in the models proposed by Grason [16] and ourselves, it is the competition between the line tension and the elastic penalty that determines the crystal morphology. Hence, while the theories proposed by Meng et al., Köhler et al., Grason, and us all assume the formation of a ribbon out of a disk-shaped crystal, the morphology of our crystals appears to be determined by the interplay between line tension and bending $[9,10,16]$. The main difference is that in our theory we explicitly assume a hybrid shape for the crystal, while Grason determines a rectangular crystal, at which point an isotropy between the width and length leads to a lower free energy. Both lead to the same scaling for the largest isotropic domain size.

Note that, although they are based on different underlying assumptions, the competition between chemical potential and bending energy leads to a scaling of the isotropic domain size of $a \sim R(\rho \Delta \mu / Y)^{1 / 4}$, while the competition between between line tension and bending energy leads to $a \sim R(\gamma / R Y)^{1 / 5}$.

A similar analysis is also possible for the average ribbon widths. Like the optimal circular domain diameter, the ribbon width is also expected to take on an optimal value, $w_{\text {eq }}$. However, this optimal width scales differently for a fixed area ribbon and a ribbon whose length is unbounded [16]. The latter case can be thought of as a single ribbon in a grandcanonical ensemble. Since in our simulations the total number 

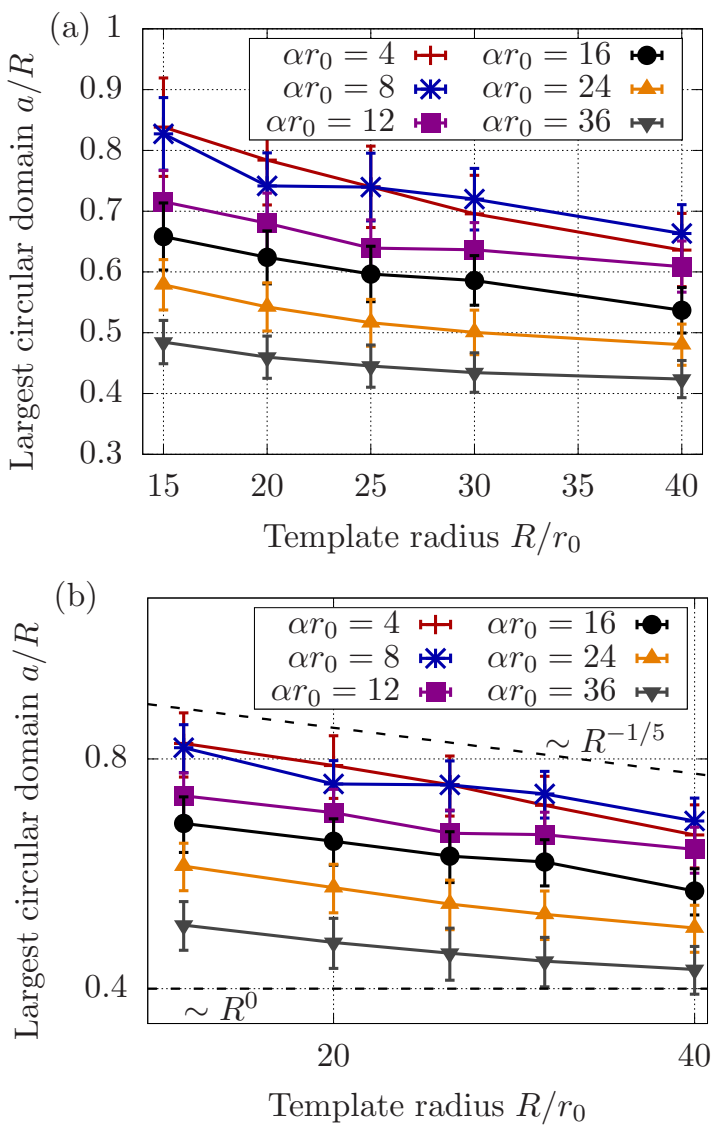

FIG. 6. Scaling of the critical isotropic domain diameter $a$ with the template radius $R$ for a constant area coverage of $40 \%$. Both the linear (a) and log-log scale (b) reveal that $a$ decreases with increasing $R$ and decreases with increasing $\alpha$. Note that the scaling with $R$ appears sublinear. The error bars represent the sampled standard error.

of particles is fixed but the particles are free to exchange between crystalline regions, it is of interest to determine the exact scaling of $w_{\text {eq }}$ with $R$ and $\alpha$. For unbounded ribbons $w_{\text {eq }} \sim R$, while for a constant area ribbon $w_{\text {eq }} \sim R^{4 / 5}[16]$. Details of our calculations are given in Appendix D. We find that within our parameter range, the equilibrium ribbon width scales sublinearly with $R$, following a trend similar to the largest isotropic crystal diameter. Hence, we conclude that our crystals are closer to the fixed area limit than the free boundary limit.

From Fig. 6 it is not only clear that the optimal circular domain size increases with $R$, it also shows that the largest isotropic domain size decreases with increasing $\alpha$, at least beyond the onset of ribbons at $\alpha r_{0} \geqslant 8$. This is qualitatively consistent with either model $[9,10,16]$. In Fig. 7, we plot the observed largest domain diameter divided by the scaling argument presented by Meng et al. [9] [Fig. 7(a)] and the heterogeneous nucleation model of Appendix D [Fig. 7(b)].

For $\alpha r_{0} \leqslant 6$, where the crystal incorporates point defects, the domain size appears to be independent of $\alpha r_{0}$. This makes sense, as the largest isotropic domain will only depend on the number of particles in the crystal, which, for our simulation setup, scales linearly with $R$. Since the scaling of the largest domain size with $\alpha$ is clearly not relevant in this regime, we
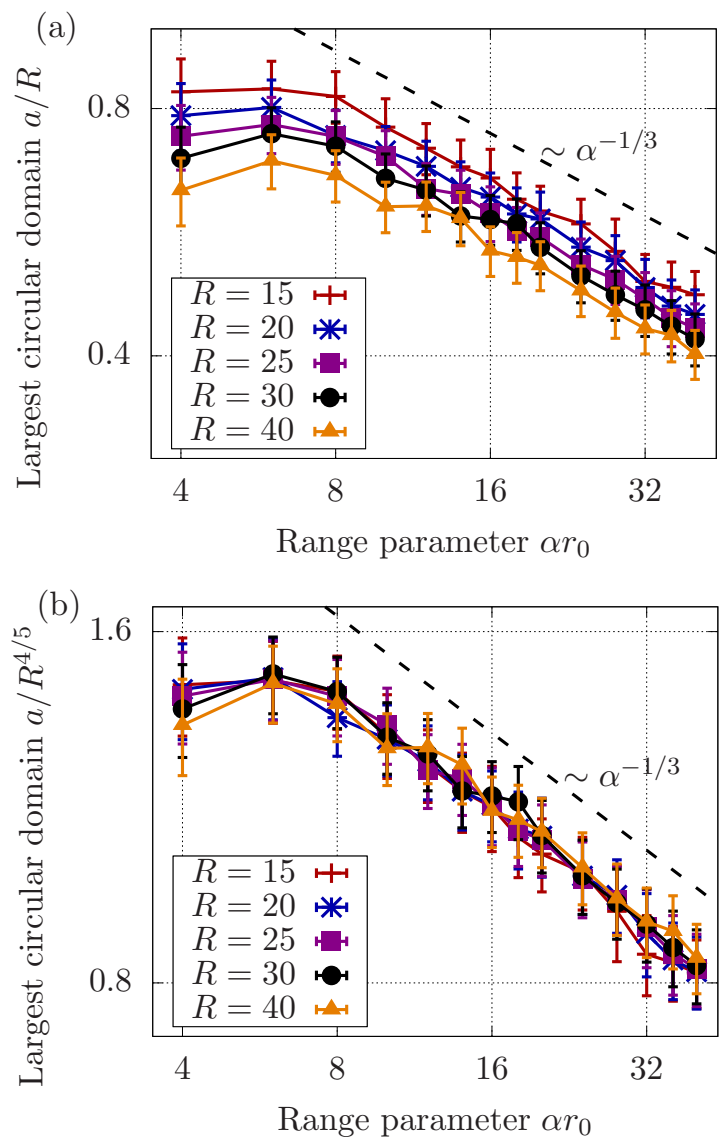

FIG. 7. Scaling of the critical isotropic domain size $a$ with the potential shape parameter $\alpha$ (a) at a constant area coverage of $40 \%$. Dividing out $R^{4 / 5}$ collapses all data onto one line (b). The scaling converges to $a \sim \alpha^{-0.33}$ (dashed line) for large $\alpha$. The transition from incorporated defects to ribbons at around $\alpha r_{0}=8$ is apparent from the different scaling with $\alpha$. The error bars represent the sampled standard error.

omit the data for $\alpha r_{0}<8$ in the following analysis of the largest isotropic domain size.

Note that the data presented in Fig. 7(b) collapse onto a single curve, giving a strong indication that, in the parameter range to which we have access, two-stage nucleation is the main pathway for ribbon formation, and that their morphology follows from the competition between line tension and elastic stress as Grason's [16] and our own competing theories imply, rather than the chemical potential and the elastic stress as put forward by Meng et al. and Köhler et al. [9,10]. For $\alpha r_{0} \geqslant 8$, fitting a power law gives us access to the scaling exponent associated with $\alpha$ of $(-0.332 \pm 0.005)$. Combining the scaling exponents for $\alpha$ and that of $(0.83 \pm 0.06)$ for $R$ quantifies our observed scaling of $a \sim R^{0.83} \alpha^{-0.33}$. The heterogeneous nucleation model predicts, with our empirical scaling of $Y$ and $\gamma$ with $\alpha$, that the scaling exponent for $\alpha$ should be $(-0.41 \pm 0.02)$, whereas the scaling argument of Meng et al. and Köhler et al. leads to a scaling exponent for $\alpha$ of $(-0.51 \pm 0.02)[9,10]$. Again, the heterogeneous nucleation, line-tension-driven model seems to better fit our observations, although the scaling with the interaction range is certainly 
less convincing than the scaling with the spherical template radius $R$.

\section{DISCUSSION AND CONCLUSION}

We performed Langevin dynamics simulations of Morse particles of diameter $r_{0}$ on spherical surfaces of varying radius. We can tune the range of interaction between the particles with a parameter $\alpha$, where larger values of $\alpha$ represent shorter ranges of attraction. We only consider a single interaction strength $\epsilon=4 k_{B} T$, at which we obtained well-equilibrated crystals. Below a critical $\alpha r_{0} \leqslant 6$, the crystals that form are disklike and incorporate dislocations and point defects. Above this critical value, the crystals instead exhibit smaller, defectfree, circlelike nuclei with protruding, ribbonlike structures. This provides a confirmation of the suggestion put forward in previous works $[9,10]$, namely that the formation of the ribbons is indeed driven by the elastic instability.

Although in the present work we have limited ourselves to positive, constant Gaussian curvature, we hypothesize that for a general surface of nonzero curvature, the onset of the instability from isotropic disklike crystals to an anisotropic crystal should still be governed by the same physics. After all, although the exact form of the elastic penalty will depend on the shape of the template, the energy penalty still scales as the square of the radius of curvature, and hence it is independent of the sign of the curvature of the surface [16]. The shape of the anisotropic crystals is, however, difficult to predict, especially for surfaces with nonconstant curvatures.

We quantified the scaling of the size of isotropic domains as a function of both the template radius $R$ and the range parameter $\alpha$, and we find that the largest circular domain diameter scales as $a \sim R^{(0.83 \pm 0.06)} \alpha^{(-0.33 \pm 0.01)}$. This is close to, but not consistent with, scalings proposed in the literature that assume the critical isotropic domain size follows from the global minimum in the free energy due to the elastic penalty $[9,10]$. Rather, we find that the scaling is more consistent with a heterogeneous nucleation model, where the transition is not dictated by the global minimum in the free energy but rather by the fact that for a sufficiently large circular domain, continued growth as a ribbon is energetically more favorable than as a circle because the line tension becomes negligible with respect to the elastic penalty. However, as we varied both parameters

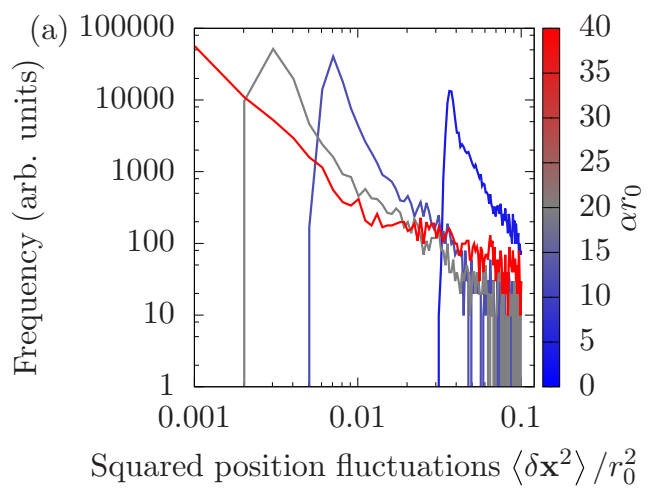

through at most one decade, further studies on larger crystals are required to determine the full parameter range over which the model is valid.

Finally, more generally, our findings indicate that, in addition to its well-known influence on the number of local minima and liquid phase stability in free space [13,14,30,31], the range of attraction also influences the morphology of the formed crystal on a curved surface. This has important consequences for, e.g., the formation of virus capsids, whose building blocks interact through short-ranged interactions [32] but are typically modeled by a long-ranged Lennard-Jones potential [33].

\section{ACKNOWLEDGMENTS}

We are grateful to Mike Hagan, Greg Grason, Jayson Paulose, Vinothan Manoharan, and Wouter Ellenbroek for valuable discussions, and Thijs van der Heijden for critical proof-reading. Furthermore, we thank the reviewers for their useful suggestions for improving the manuscript. We acknowledge the HFSP for funding under Grant No. RGP0017/2012.

S.P. and P.v.d.S. conceived the idea. S.P. and P.v.d.S. developed the theory for heterogeneous nucleation. S.P. and P.v.d.S. wrote the manuscript. S.P. performed the simulations. G.B. assisted in calculating the effective spring constants.

\section{APPENDIX A: EXTRACTING EFFECTIVE SPRING CONSTANTS}

To estimate the scaling of Young's modulus, we require the effective spring constant $\kappa$ that keeps the particles in place in the crystal. For a single-particle pair, this can be found analytically by harmonically approximating the pair potential around the minimum. For the case of the Morse potential, this leads to $\kappa=2 \alpha^{2} \epsilon$. However, this simple approximation does not take into account collective effects. Therefore, we extract effective spring constants from our simulation data.

From our simulation trajectories, we determine a running average of the particle positions $\mathbf{x}$ by applying an exponentially weighted average to obtain for a time step $t_{n}$ the average $\mathbf{x}_{a}\left(t_{n}\right)=(1-\xi) \mathbf{x}\left(t_{n}\right)+\xi \mathbf{x}_{a}\left(t_{n-1}\right)$. The parameter $\xi$ controls how fast the past positions are "forgotten." At each time step, we determine the squared deviation of the particles from the average from the previous frame, $\delta \mathbf{x}^{2}\left(t_{n}\right):=\left[\mathbf{x}\left(t_{n}\right)-\right.$ $\left.\mathbf{x}_{a}\left(t_{n-1}\right)\right]^{2}$. This serves as an approximation of the squared

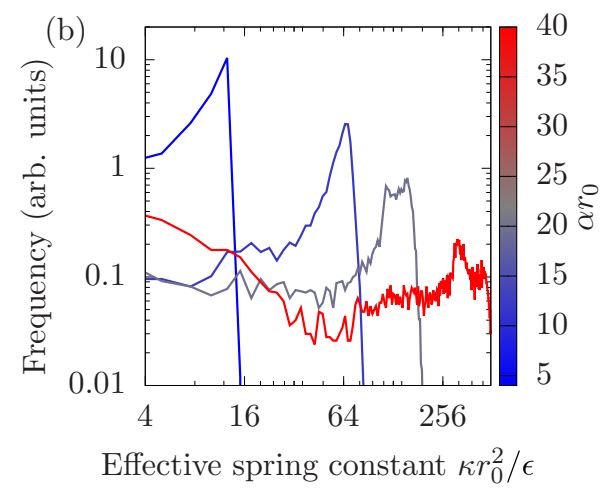

FIG. 8. Distributions of the squared fluctuations in the particle positions about their lattice site (a) and of the effective spring constant $\kappa=2 k_{B} T /\left\langle\delta \mathbf{x}^{2}\right\rangle$ (b), both for $R=40 r_{0}$ and an area coverage of $40 \%$. For increasing $\alpha$ the peak in $\langle\delta \mathbf{x}\rangle^{2}$ shifts to the left and, consistently, the peak in $\kappa$ shift to the right. 


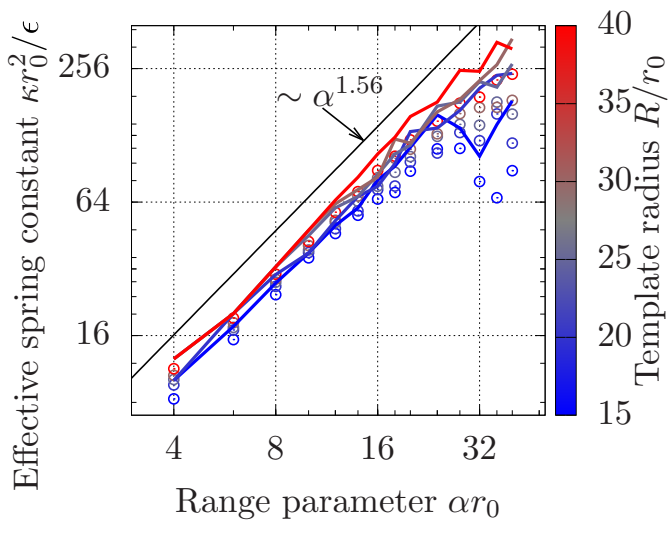

FIG. 9. Extracted modal (lines) and average (symbols) of the effective spring constant distributions from Fig. 8. The data for $R=$ $40 r_{0}$ best follow the power law $\kappa \sim \alpha^{1.56}$ with the asymptotic standard error of the fit being 0.05 .

deviation of the particle from its average lattice site while taking into account that the lattice site might drift in time. Typical squared displacement values we find are of the order of $0.05 r_{0}^{2}$. We empirically tuned $\xi$ to a value of 0.9 at which the fast motions about the average lattice site are suppressed.

This produces for each particle a time trace of the quantity $\delta \mathbf{x}^{2}$, which can be averaged in time to obtain $\left\langle\delta \mathbf{x}^{2}\right\rangle$. If we assume particles are bound to their lattice site by a harmonic spring, the effective spring constant then follows from equipartition as $\frac{1}{2} \kappa\left\langle\delta \mathbf{x}^{2}\right\rangle=k_{B} T$. In Fig. 8, we present distributions for the squared deviation and effective spring constant for four values of $\alpha$.

In Fig. 9, we present the effective spring constants for all parameter values. Note that for very large $\alpha$ the scaling breaks down. This is because the script used to determine the peak in the histogram simply determines the numerical maximum as an approximation for the mean. This breaks down for noisy and particularly wide distributions, which we obtain for large $\alpha$, as can be seen from the gray and red distributions in Fig. 8(b).

\section{APPENDIX B: DETERMINING THE CHEMICAL POTENTIAL}

To determine the chemical potential of a particle in the crystal, we determine the free-energy difference between the original crystal of $N$ Morse particles and a hybrid MorseEinstein crystal in which the particle that is most representative of the bulk of the crystal is replaced by an Einstein particle tethered to its initial lattice site with a harmonic spring. To determine the most representative particle, we apply the analysis to extract the largest isotropic domain size, which we discuss in Appendix D. This analysis finds the particle that is the farthest away from all edge particles, which is the best available representative of the bulk.

We set the spring constant to $k=50 \epsilon / r_{0}^{2}$, at which the Einstein particle and the original Morse particle have comparable root-mean-square deviations from the average lattice site, calculated following the procedure detailed in Appendix A. We apply Bennett's acceptance criterion [27] to the two different cases, whose total potential energies are

$$
\begin{aligned}
U_{0} & =\sum_{i=0}^{N-1} \sum_{j>i}^{N-1} U\left(r_{i j}\right)+\sum_{i=0}^{N} U\left(\mathbf{r}_{i N}\right), \\
U_{1} & =\sum_{i=0}^{N-1} \sum_{j>i}^{N-1} U\left(r_{i j}\right)+U_{E}\left(\mathbf{x}_{N}\right)
\end{aligned}
$$

with

$$
\begin{aligned}
r_{i j} & :=\left\|\mathbf{x}_{i}-\mathbf{x}_{j}\right\|, \\
U(r) & =\left[U_{M}(r)-U_{M}\left(r_{c}\right)\right] H\left(r_{c}-r\right), \\
U_{M}(r) & :=\epsilon\left[e^{-2 \alpha\left(r-r_{0}\right)}-2 e^{-\alpha\left(r-r_{0}\right)}\right],
\end{aligned}
$$

and

$$
U_{E}\left(\mathbf{x}_{N}\right)=\frac{1}{2} \kappa\left[\mathbf{x}_{N}-\mathbf{x}_{N}(0)\right]^{2}
$$

Here, $U_{0}$ is the original truncated, shifted Morse potential $U$ with cutoff distance $r_{c}$, well depth $\epsilon$, and range parameter $\alpha$ acting on all $N$ particles with diameter $r_{0}$, while $U_{1}$ is the original Morse potential acting on $N-1$ particles combined with an "Einstein potential" $U_{E}$ applied to the $N$ th particle. $H(x)$ is the Heaviside function with $H(x)=1$ for $x>0$ and 0 otherwise. The Einstein potential is, of course, a harmonic spring with spring constant $\kappa$ that attaches the $N$ th particle to its initial position $\mathbf{x}_{N}(0)$. Finally, $r_{i j}$ is the scalar distance between particles $i$ and $j$, located at positions $\mathbf{x}_{i}$ and $\mathbf{x}_{j}$.

We apply Bennett's acceptance criterion [27] with energy offset $C=2.4 \epsilon$ and sample

$$
e^{-\beta\left(F_{1}-F_{0}-C\right)}=\frac{\left\langle f\left[\beta\left(U_{1}-U_{0}-C\right)\right]\right\rangle_{0}}{\left\langle f\left[\beta\left(U_{0}-U_{1}+C\right)\right]\right\rangle_{1}},
$$

where $\beta$ is the reciprocal thermal energy $1 / k_{B} T, f(x)$ is the Fermi function $f(x)=1 /[1+\exp (x)]$, and the subscripts 0 and 1 mean that the average is obtained by sampling the potential energy function $U_{0}$ and $U_{1}$, respectively. The value of $C$ corresponds roughly to the average potential energy difference between the two potential energy functions, $C=\left\langle U_{1}\right\rangle_{1}-\left\langle U_{0}\right\rangle_{0}$. We determined this value for $\alpha=16 / r_{0}$ but applied it for all $\alpha$. For varying $\alpha$, the averages $\left\langle U_{1}\right\rangle$ and $\left\langle U_{0}\right\rangle$ do differ significantly, but the method remains usable for this one fixed value of $C$.

Applying Bennett's acceptance ratio means that we generate trajectories corresponding to both $U_{0}$ and $U_{1}$, and we average $f\left[\beta\left(U_{1}-U_{0}-C\right)\right]$ over the trajectory generated by $U_{0}$, while we average $f\left[\beta\left(U_{0}-U_{1}+C\right)\right]$ over the trajectory generated by $U_{1}$. This can then be converted straightforwardly into an estimate for the free-energy difference between the Morse and the Morse-Einstein crystal. The excess chemical potential of the Einstein particle $\mu_{E}^{\mathrm{ex}}$ can be calculated analytically from the partition function and is $\mu_{E}^{\mathrm{ex}}=k_{B} T \ln \left(\kappa A / 2 \pi N k_{B} T\right)$, where $\kappa$ is the spring constant, $A$ is the spherical template area, $N$ is the number of particles on the template, and $k_{B} T$ is the thermal energy. Hence, the free-energy difference can directly be converted into an excess chemical potential by 

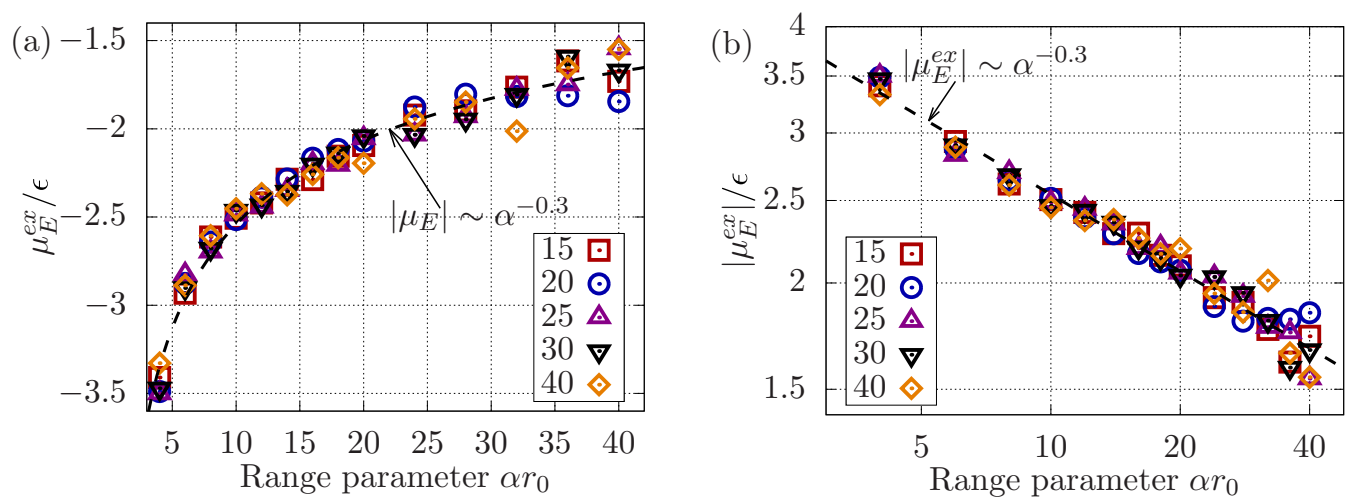

FIG. 10. Calculated excess chemical potential for $N$ Morse particles in linear (a) and log-log (b) scale. The symbols denote different spherical template radii. Note that increasing $\alpha$ leads to a higher chemical potential (lower absolute value), and the scaling appears to be independent of the spherical template radius.

adding $\mu_{E}^{\mathrm{ex}}$, which we present in Fig. 10. The free-energy difference appears to scale as $\mu_{E}^{\mathrm{ex}} \sim \alpha^{-0.52}$, independent of the spherical template radius.

\section{APPENDIX C: EXTRACTING THE LARGEST DOMAIN SIZE}

To analyze the ribbon widths, a series of steps is required. Each of them is explained in detail here. First, we construct a network of nearest neighbors. Particles are considered nearest neighbors if their distance is less than $1.3 r_{0}$ apart. This distance coincides with the minimum after the first peak in the pair correlation function. From this information, we can identify "edge particles," i.e., particles that do not have six nearest neighbors.

With this information, we can compute for all particles that are not edge particles the distance to the nearest edge particles. The largest of these distances we take as an estimate for the largest isotropic domain size, as it represents the largest possible circle diameter that fits inside the crystal. This assumption breaks down for $\alpha$ sufficiently small to allow for incorporation of defects inside the lattice, rather than at the edge. This is observed for $\alpha \leqslant 6$ in Fig. 3(a). In Fig. 11, we present snapshots of the different stages of analysis. With the aforementioned steps, we obtain a value for the largest circular domain diameter for each combination of spherical template radius $R$ and potential range parameter $\alpha$.

\section{APPENDIX D: SCALING OF RIBBON WIDTHS}

To determine how the average ribbon widths scale as a function of $\alpha$ and $R$, we apply the same scheme as described in Appendix $C$ to extract the largest domain size. However, now, instead of only extracting the largest distance, we extract all local largest distances. In other words, we determine for each particle if the shortest distance from that particle to the edge is larger than that distance for any of its neighbors. If so, this particle represents a local maximum in terms of distance to the edge, and twice this distance is an approximation for the local width of the ribbon. Averaging all these quantities leads to an average ribbon width.

The interest in the average ribbon width comes from the fact that the optimal ribbon width scales differently in the constant area limit, where the number of particles per ribbon is fixed, and the free boundary limit, in which the ribbon length can grow indefinitely while the width adopts an optimal value $w_{\text {eq }}$. In the case of fixed area, $w_{\text {eq }} \approx$ $2 R(\gamma / R Y)^{1 / 5} \sim R^{4 / 5} / Y^{1 / 5}$, while for the free boundary limit it is $w_{\text {eq }} \approx(288 \Delta \mu \rho / Y)^{1 / 4} R \sim R / Y^{1 / 4}$ [16]. Hence, it is interesting to see whether our simulations agree with either of
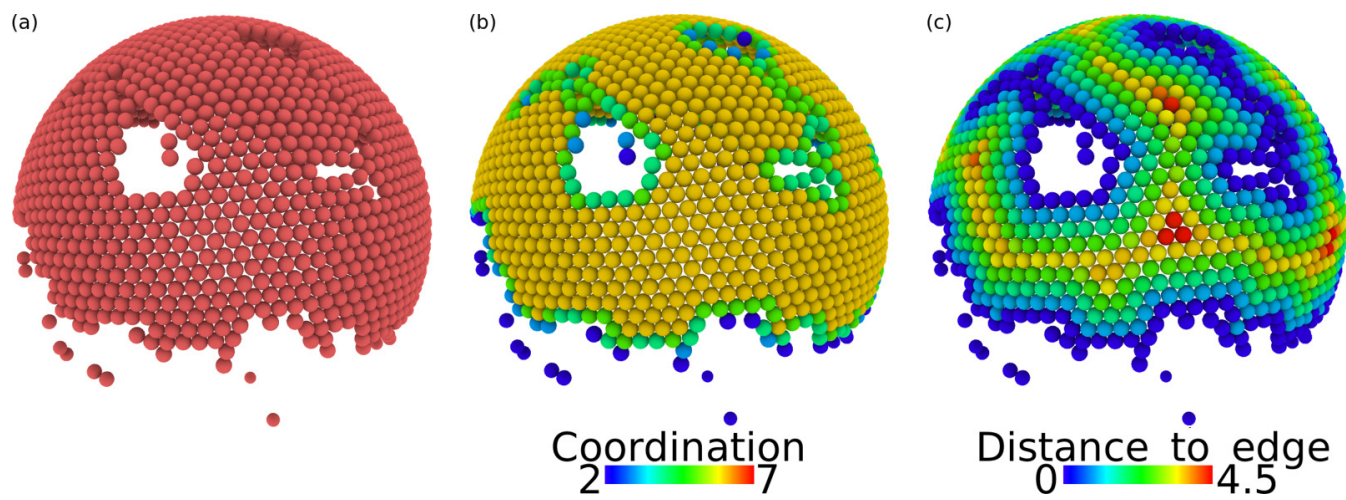

FIG. 11. Different analysis stages of the same snapshot (a) obtained for $\alpha=20 / r_{0}, R=15 r_{0}$ at area coverage $\phi=0.4$. (b) The coordination $n_{c}$ (number of nearest neighbors) identifies bulk particles $\left(n_{c}=6\right)$ and edge particles $\left(n_{c} \neq 6\right)$. (c) We calculate for each bulk particle the shortest distance to the edge particles (color-coded, units of $r_{0}$ ). 

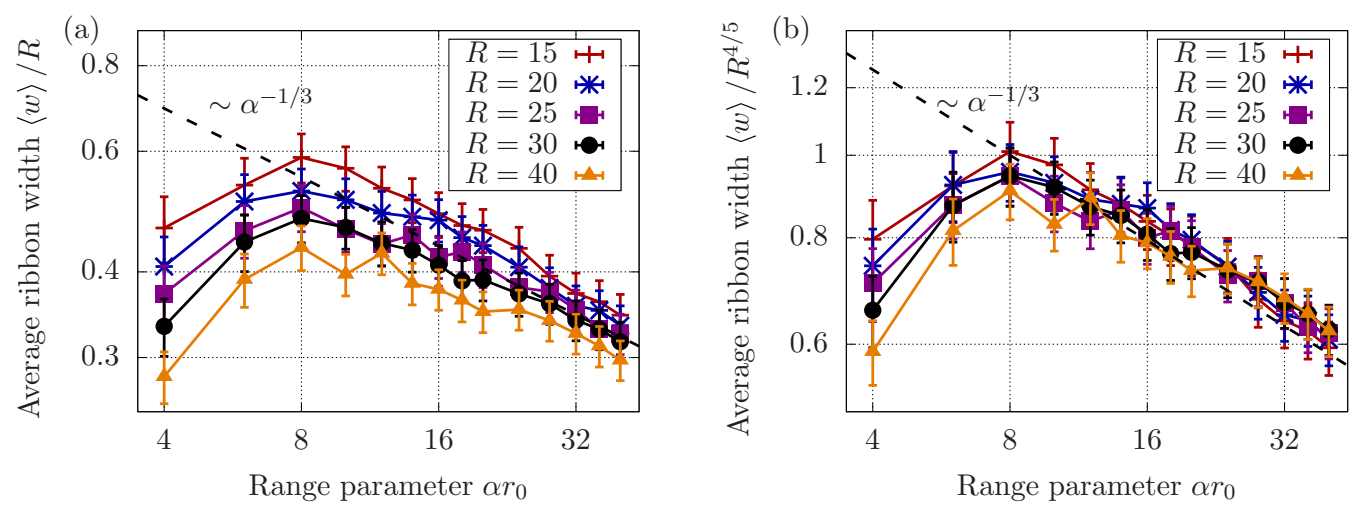

FIG. 12. Scaling of the average ribbon width. Dividing out the scaling expected for the free boundary limit (a) reveals that out data do not match this theory perfectly. In (b) we divide the expected scaling for the constant area model and find a very good agreement.

these predictions. After all, although we have a fixed number of particles on the spherical template, the number of particles per ribbon is not necessarily constant. Note that both scalings are qualitatively the same and quantitatively close. We plot the average widths in Fig. 12.

From Fig. 12 it is clear that our data match the qualitative prediction of either limit well. If we divide out the free boundary limit scaling, we find that the curves do not all collapse on the same curve, unlike when we divide out the constant area scaling. Hence, we conclude that our data are best described by the constant area limit. Finally, note that the average widths scale the same way with $\alpha$ as the largest circular domain sizes $a$ (Fig. 5 in the main text), namely as $\alpha^{-1 / 3} R^{4 / 5}$.
[1] A. D. Dinsmore, M. F. Hsu, M. G. Nikolaides, M. Marquez, A. R. Bausch, and D. A. Weitz, Science 298, 1006 (2002).

[2] F. Sausset, G. Tarjus, and D. R. Nelson, Phys. Rev. E 81, 031504 (2010).

[3] C. J. Burke, B. L. Mbanga, Z. Wei, P. T. Spicer, and T. J. Atherton, Soft Matter 11, 5872 (2015).

[4] C. J. Burke and T. J. Atherton, arXiv:1605.09478.

[5] A. R. Bausch, M. J. Bowick, A. Cacciuto, A. D. Dinsmore, M. F. Hsu, D. R. Nelson, M. G. Nikolaides, A. Travesset, and D. A. Weitz, Science 299, 1716 (2003).

[6] P. Lipowsky, M. J. Bowick, J. H. Meinke, D. R. Nelson, and A. R. Bausch, Nat. Mater. 4, 407 (2005).

[7] T. Einert, P. Lipowsky, J. Schilling, M. J. Bowick, and A. R. Bausch, Langmuir 21, 12076 (2005).

[8] W. T. M. Irvine, V. Vincenzo, and P. M. Chaikin, Nature (London) 468, 947 (2010).

[9] G. Meng, J. Paulose, D. R. Nelson, and V. N. Manoharan, Science 343, 634 (2014).

[10] C. Köhler, R. Backofen, and A. Voigt, Phys. Rev. Lett. 116, 135502 (2016).

[11] S. Schneider and G. Gompper, Europhys. Lett. 70, 136 (2005).

[12] C. Majidi and R. S. Fearing, Proc. R. Soc. London, Ser. A 464, 1309 (2008).

[13] J. P. K. Doye, D. J. Wales, and R. S. Berry, J. Chem. Phys. 103, 4234 (1995).

[14] J. P. K. Doye and D. J. Wales, J. Phys. B 29, 4859 (1996).
[15] S. Paquay, H. Kusumaatmaja, D. J. Wales, R. Zandi, and P. van der Schoot, Soft Matter 12, 5708 (2016).

[16] G. M. Grason, J. Chem. Phys. 145, 110901 (2016).

[17] D. Kashchiev, Nucleation (Butterworth-Heinemann, Oxford, UK, 2000).

[18] D. T. Wu, Solid State Phys. 50, 37 (1996).

[19] A. A. Chernov, J. Cryst. Growth 24, 11 (1974).

[20] L. R. Gomez, N. A. Garcia, V. Vitelli, J. Lorenzana, and D. A. Vega, Nat. Commun. 6, 6856 (2015).

[21] S. Plimpton, J. Comput. Phys. 117, 1 (1995).

[22] H. C. Andersen, J. Comput. Phys. 52, 24 (1983).

[23] S. Paquay and R. Kusters, Biophys. J. 110, 1226 (2016).

[24] V. Vitelli, J. B. Lucks, and D. R. Nelson, Proc. Natl. Acad. Sci. USA 103, 12323 (2006).

[25] D. Squire, A. Holt, and W. Hoover, Physica 42, 388 (1969).

[26] H. Löwen, J. Phys.: Condens. Matter 2, 8477 (1990).

[27] C. H. Bennett, J. Comput. Phys. 22, 245 (1976).

[28] A. Stukowski, Modell. Simul. Mater. Sci. Eng. 18, 015012 (2010).

[29] C. Qiao, Ph.D. thesis, Brandeis University, 2016.

[30] J. P. K. Doye and D. J. Wales, Science 271, 484 (1996).

[31] M. H. J. Hagen, E. J. Meijer, G. C. A. M. Mooij, D. Frenkel, and H. N. W. Lekkerkerker, Nature (London) 365, 425 (1993).

[32] V. A. Parsegian, Van der Waals Forces (Cambridge University Press, Cambridge, 2005).

[33] R. Zandi, D. Reguera, R. F. Bruinsma, W. M. Gelbart, and J. Rudnick, Proc. Natl. Acad. Sci. USA 101, 15556 (2004). 